\title{
FATORES ASSOCIADOS AO DESEMPENHO DE SERVIÇOS DA ATENÇÃO PRIMÁRIA À SAÚDE
}

\section{Factors associated with the performance of primary health care services \\ Factores asociados con el rendimiento de los servicios de la atención primaria de salud}

\author{
Anna Gabryela Sousa Duarte iD \\ Universidade Federal de São João del-Rei - UFSJ - Divinópolis (MG) - Brasil \\ Tarcísio Laerte Gontijo (iD \\ Universidade Federal de São João del-Rei - UFSJ - Divinópolis (MG) - Brasil \\ Eliete Albano de Azevedo Guimarães iD \\ Universidade Federal de São João del-Rei - UFSJ - Divinópolis (MG) - Brasil
}

Ricardo Bezerra Cavalcante iD

Universidade Federal de Juiz de Fora - UFJF - Juiz de Fora (MG) - Brasil

Vinícius Silva Belo iD

Universidade Federal de São João del-Rei - UFSJ - Divinópolis (MG) - Brasil

\section{George Sobrinho Silva iD}

Universidade Federal dos Vales do Jequitinhonha e Mucuri - UFVJM - Diamantina (MG) - Brasil

\section{RESUMO}

Objetivo: Avaliar o desempenho de equipes de Saúde da Família (eSF) e equipes de Atenção Básica (eAB) sobre a presença e extensão de atributos da Atenção Primária à Saúde (APS) através da visão dos usuários. Métodos: Trata-se de um estudo observacional transversal realizado em um município da região centro-oeste de Minas Gerais (MG) entre janeiro e junho de 2016 com amostra representativa de 384 usuários assistidos por eSF e eAB. Os dados foram obtidos por meio da aplicação do instrumento Primary Care Assessment Tool (PCATool-Brasil) - usuários adultos. Inicialmente, calculou-se o escore de cada atributo/componente e os escores essencial e geral. Utilizou-se a modelagem de análise multivariada para identificar variáveis associadas à avaliação satisfatória (escore $\geq 6,6$ ) da APS. Resultados: Os resultados apontam que a maioria dos atributos/ componentes da APS não está devidamente implantado no cenário em questão. Identificou-se, ainda, na análise multivariada, que a avaliação de usuários da eSF (Odds Ratio: 2,09) e de pessoas com até oito anos de estudo (Odds Ratio: 1,94) está associada à avaliação satisfatória da APS. Conclusão: Na visão dos usuários, a eSF tem maior potencial para a presença e extensão dos atributos/componentes da APS, reforçando, assim, o papel de destaque dessa estratégia como principal arranjo organizacional de APS no cenário nacional.

Descritores: Avaliação em Saúde; Atenção Primária à Saúde; Saúde da Família.

\section{ABSTRACT}

Objective: To assess the performance of Family Health teams (equipes de Saúde da Familia - eSF) and Primary Care teams (equipes de Atenção Básica - eAB) in relation to the presence and extension of attributes of Primary Health Care (PHC) from users' point of view. Methods: This is an observational cross-sectional study conducted in a municipality in the Midwest region of Minas Gerais (MG), Brazil, between January and June 2016 with a representative sample of 384 users assisted by eSF and eSF. Data were obtained through the application of the Primary Care Assessment Tool (PCATool-Brazil) - adult users. First, the score for each attribute/component and the essential and general scores were calculated. Multivariate analysis modeling was used to identify variables associated with satisfactory assessment (score $\geq 6.6$ ) of PHC. Results: The results indicate that most

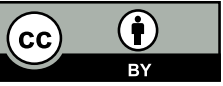


attributes/components of PHC are not properly implemented in the scenario analyzed. The multivariate analysis also showed that the assessments made by users of eSF (Odds Ratio: 2.09) and people with up to eight years of study (Odds Ratio: 1.94) were associated with a satisfactory assessment of PHC. Conclusion: According to the users' point of view, eSF have a greater potential for the presence and extension of attributes/components of PHC, thus reinforcing the important role of this strategy as the main organizational arrangement of PHC in the national scenario.

Descriptors: Health Evaluation; Primary Health Care; Family Health.

\section{RESUMEN}

Objetivo: Evaluar el rendimiento de los equipos de Salud de la Familia (eSF) y los equipos de la Atención Básica (eAB) sobre la presencia y extensión de los atributos de la Atención Primaria de Salud (APS) a través de la percepción de los usuarios. Métodos: Se trata de un estudio observacional transversal realizado en un municipio de la región centro-oeste de Minas Gerais (MG), Brasil, entre enero y junio de 2016 con una muestra representativa de 384 usuarios asistidos por el eSF y el eAB. Se han obtenido los datos a través de la aplicación del instrumento Primary Care Assessment Tool (PCATool-Brasil) - usuarios adultos. A principio, se ha calculado la puntuación de cada atributo/componente y las puntuaciones esencial y general. Se utilizó el análisis multivariado para identificar las variables asociadas con la evaluación satisfactoria (puntuación $\geq 6,6)$ de la APS. Resultados: Los resultados señalan que la mayoría de los atributos/componentes de la APS no se ha implantado correctamente en el escenario en cuestión. Se ha identificado, aún, en el análisis multivariado, que la evaluación de los usuarios de la eSF (Odds Ratio: 2,09) y de las personas con hasta ocho años de escolaridad (Odds Ratio: 1,94) se asocia con la evaluación satisfactoria de la APS. Conclusión: En la percepción de los usuarios, el eSF tiene mayor potencial para la presencia y extensión de los atributos/componentes de la APS, reforzando, de esa manera, el papel de destaque de esa estrategia como el principal ajuste organizacional de la APS para el escenario nacional.

Descriptores: Evaluación en Salud; Atención Primaria de Salud; Salud de la Familia.

\section{INTRODUÇÃO}

A Atenção Primária à Saúde (APS) possui grande importância na operacionalização dos sistemas de saúde. Estrutura-se como principal porta de entrada dos usuários no sistema, ordenando as ações e serviços disponibilizados pela Rede de Atenção à Saúde (RAS) ${ }^{(1)}$. A abrangência das ações ofertadas, a acessibilidade, a continuidade e coordenação do cuidado, além da responsabilização sobre determinado território, diferencia a APS de outros tipos de serviços assistenciais ${ }^{(2)}$.

No Brasil, a expansão e consolidação da APS se deram, principalmente, por meio da Estratégia Saúde da Família (ESF), considerada o principal arranjo de organização da APS no país. Em 2018, a cobertura nacional da ESF era de $59,7 \%$ da população, contando com 39.872 equipes de Saúde da Família (eSF) distribuídas em 5.402 municípios brasileiros ${ }^{(3)}$. O aumento progressivo de cobertura populacional pela ESF foi acompanhado de melhorias na estrutura física dos serviços e da qualificação dos profissionais e gestores da APS ${ }^{(4)}$.

A ESF tem contribuído para ampliação do acesso, prevenção de agravos, busca ativa de casos, educação em saúde, assistência domiciliar e aumento do número de atendimentos relacionados à saúde das mulheres e das crianças. Tudo isto tem produzido melhorias em indicadores de saúde, como a redução de nascimentos com baixo peso, mortalidade infantil e internações hospitalares ${ }^{(5)}$.

É importante ressaltar que existem, além da ESF, outros arranjos organizacionais de assistência à APS em desenvolvimento no país. Segundo a Política Nacional de Atenção Básica (PNAB) vigente, todos esses outros arranjos são denominados de equipes de Atenção Básica $(\mathrm{e} A B)$ e serão reconhecidos desde que respeitem os princípios e diretrizes do Sistema Único de Saúde (SUS) ${ }^{(1)}$. A maioria das eAB está voltada para a assistência à doença em seus aspectos individuais e biológicos e para a priorização da demanda espontânea e/ou encaminhada de outros serviços, sem ênfase às ações programáticas ${ }^{(6)}$.

Em geral, a ESF tem sido mais bem avaliada que as eAB no que diz respeito à equidade, integralidade da atenção e ações programáticas, desempenho, trabalho multidisciplinar, orientação familiar e comunitária, acolhimento, vínculo e humanização(5).

AAPS, independente do arranjo organizacional adotado, deve ser orientada por atributos essenciais e derivados ${ }^{(7)}$. Os atributos essenciais compreendem o acesso de primeiro contato, a longitudinalidade, a integralidade e a coordenação. Já os derivados envolvem o enfoque familiar, a orientação comunitária e a competência cultural( ${ }^{(7,8)}$. A presença e extensão desses atributos qualificam a APS, aumentando oportunamente a interação com usuários e comunidade ${ }^{(7,9)}$. 
A avaliação da orientação de um serviço à APS, através da presença e extensão de seus atributos, visa ao melhor desempenho e qualificação dos serviços, à consolidação das ações implementadas e à capacidade de resposta às necessidades de saúde da população(10,11).

Dado o papel de destaque da APS nos sistemas de saúde e os seus diferentes arranjos assistenciais, ainda presentes, faz-se necessário realizar avaliações que visem a analisar o desempenho desses diferentes arranjos ${ }^{(12)}$. Além disso, a PNAB destaca que toda Unidade Básica de Saúde (UBS), independente do tipo de equipe implantada, deve monitorar e avaliar o desempenho de suas ações por meio da satisfação de seus usuários. Porém esta não é uma prática realizada com frequência ${ }^{(1,13)}$.

A avaliação da APS pode impactar na promoção da saúde da população, bem como na construção e revisão de políticas públicas saudáveis como referência central para a ação institucionalizada em saúde, fortalecendo estruturas e processos comprometidos nessa vertente. Nesse sentido, faz-se necessário ampliar o entendimento da promoção da saúde no contexto da atenção primária como referência para todo o processo de trabalho. Por isso, a avaliação dos serviços de saúde têm ganhado protagonismo no aprimoramento de políticas públicas e na reorientação das ações em saúde, aperfeiçoando o desempenho do SUS ${ }^{(14)}$.

É nesse contexto que emerge este estudo, que visa a avaliar o desempenho de equipes de Saúde da Família (eSF) e equipes de Atenção Básica (eAB) sobre a presença e extensão de atributos da APS através da visão dos usuários.

\section{MÉTODOS}

Trata-se de estudo transversal realizado em um município da região centro-oeste de Minas Gerais (MG), Brasil, que possui população estimada de aproximadamente 234.937 habitantes $^{(15)}$ e conta com 42 UBS, sendo 32 com equipes de Saúde da Família (eSF) e 10 com equipes de Atenção Básica (eAB).

Participaram deste estudo usuários atendidos em UBS da área de abrangência de sua residência, tendo sido incluídas as UBS com mais de um ano de funcionamento. Nesse sentido, recrutaram-se participantes em 26 UBS, sendo 16 equipadas com eSF e $10 \mathrm{com}$ eAB. O dimensionamento amostral definiu uma população finita e um erro absoluto de $5 \%$. O resultado do dimensionamento demonstrou a necessidade da utilização de 384 participantes, que foram estratificados por tipo de UBS. Sendo assim, definiu-se uma amostra de 117 usuários assistidos por eSF e 267 usuários assistidos por eAB levando em consideração a população cadastrada em cada unidade de saúde incluída no estudo.

A coleta de dados ocorreu entre janeiro e junho de 2016. Entrevistaram-se usuários com mais de 18 anos, que estavam presentes para algum tipo de atendimento nas UBS selecionadas, em dia pré-determinado pelos pesquisadores, e que aceitaram participar do estudo. As entrevistas, realizadas por pesquisador treinado, duraram, em média, 15 minutos.

Utilizou-se o instrumento Primary Care Assessment Tool (PCATool-Brasil) - usuários adultos, versão reduzida para coleta de dados. A versão reduzida do PCATool-Brasil é validada, está disponível gratuitamente e mede a presença e extensão dos atributos da APS. É composta por 23 itens, contemplando os atributos essenciais: primeiro contato do indivíduo com o sistema de saúde (utilização e acesso); longitudinalidade; integralidade (serviços disponíveis e serviços prestados); coordenação (cuidado e sistema de informação) e os atributos derivados: enfoque familiar e orientação comunitária ${ }^{(16)}$.

Destaca-se que o PCATool-Brasil permite a comparação do grau de orientação à APS de outros modelos de atenção básica que ainda coexistem junto à ESF, permitindo identificar aspectos de estrutura e processo dos serviços que exigem reafirmação ou reformulação na busca da qualidade, tanto para o planejamento como para a execução das ações de APS ${ }^{(17)}$.

As cinco opções de resposta do instrumento são por meio de escala Likert: 'com certeza sim' (valor=4); 'provavelmente sim' (valor=3); 'provavelmente não' (valor=2); 'com certeza não' (valor=1) e; 'não sei/não lembro' $(\text { valor }=9)^{(17)}$. Também se utilizou um questionário elaborado pelos pesquisadores a respeito do perfil sociodemográfico dos participantes contendo questões relativas ao sexo, idade, escolaridade e situação conjugal.

A fim de assegurar a qualidade dos dados, realizou-se a dupla digitação, com posterior comparação dos resultados. Realizaram-se todas as tabulações e análises com auxílio do software Statistical Package for Social Sciences (SPSS), versão 20.0. Calcularam-se os escores referente a cada um dos atributos da APS presentes no PCATool-Brasil - usuários adultos, na versão reduzida. A obtenção dos escores ocorreu pela média aritmética simples dos valores das respostas dos itens que o compõe transformados em escala de 0 a 10 utilizando a seguinte fórmula: (Escore obtido - 1) x 10/3 ${ }^{(17)}$. 
Em seguida, calcularam-se os escores essencial (medido pela soma do grau de afiliação mais escore médio de cada um dos componentes que pertencem aos atributos essenciais, dividido pelo número de componentes) e geral (medido pela soma do grau de afiliação mais escore médio dos componentes que pertencem aos atributos essenciais e derivados, dividido pelo número total de componentes) da APS ${ }^{(17)}$, assim como a proporção de usuários que atribuíram altos escores $(\geq 6,6)$ para o munícipio em geral e por tipo de UBS, eSF e eAB. O grau de afiliação visa a identificar o serviço ou profissional de saúde (médico/enfermeiro) que serve como referência para o cuidado, o que não é considerado um atributo da APS, porém é utilizado no cálculo dos escores essencial e geral(17).

Para a execução das análises estatísticas, utilizou-se o teste qui quadrado, a um nível de significância de 5\%, para a comparação das proporções de avaliações dos escores essencial e geral da APS satisfatórias (escores $\geq$ $6,6)$ entre as variáveis de exposição (sexo, idade, escolaridade, situação conjugal e tipo de UBS). E, em seguida, realizou-se a análise multivariada por meio de modelos de regressão logística.

Incluíram-se na modelagem as variáveis que apresentaram valor de $p<0,25$ nas associações bivariadas. No modelo final, após a execução de procedimentos backward não automáticos, mantiveram-se aquelas com valor de $p<0,05$. Com a modelagem, obtiveram-se os valores de Odds Ratio ajustados e seus respectivos intervalos de confiança, de $95 \%$, para as variáveis selecionadas no modelo final. O ajuste do modelo final, por sua vez, foi avaliado pelo teste de Hosmer e Lemeshow.

O presente estudo obedeceu aos preceitos éticos da Resolução n. ${ }^{\circ} 466 / 2012$ e foi aprovado por Comitê de Ética em Pesquisas sob o Parecer n. ${ }^{\circ} 1.234 .728$. Todos os participantes assinaram o Termo de Consentimento Livre e Esclarecido, em conformidade com a legislação vigente.

\section{RESULTADOS}

Participaram do estudo 384 usuários, vinculados a 26 UBS, sendo 117 usuários assistidos por eSF e 267 usuários por eAB. Em relação ao perfil dos participantes, identificou-se que a maioria era do sexo feminino, possuía entre 40 e 59 anos de idade, estava em união estável e tinha até 8 anos de estudo (Tabela I).

Tabela I - Perfil sociodemográfico dos participantes segundo o tipo de Unidade Básica de Saúde. Minas Gerais, Brasil, 2016.

\begin{tabular}{lccc}
\hline Perfil sociodemográfico & $\begin{array}{c}\text { Total } \\
\mathbf{n}(\%)\end{array}$ & $\begin{array}{c}\text { Tipo de Unidade Básica de Saúde } \\
\text { eSF } \\
\mathbf{n}(\%)\end{array}$ & $\begin{array}{c}\text { eAB } \\
\mathbf{n}(\%)\end{array}$ \\
\hline Sexo & & & \\
$\quad$ Masculino & $74(19,3)$ & $22(18,8)$ & $52(19,5)$ \\
$\quad$ Feminino & $309(80,5)$ & $95(81,2)$ & $214(80,1)$ \\
$\quad$ Não informado & $1(0,2)$ & - & $1(0,4)$ \\
Idade (anos) & & & $78(29,2)$ \\
Até 40 & $127(33,1)$ & $49(41,9)$ & $112(42,0)$ \\
De 40 a 59 & $160(41,7)$ & $48(41,0)$ & $76(28,5)$ \\
60 e mais & $96(25,0)$ & $20(17,1)$ & $1(0,3)$ \\
Não informado & $1(0,2)$ & - & $116(43,4)$ \\
Situação conjugal & & & $149(55,8)$ \\
Solteiro, divorciado, viúvo & $162(42,2)$ & $46(39,3)$ & $2(0,8)$ \\
União estável & $220(57,3)$ & $71(60,7)$ & $168(63,0)$ \\
Não informado & $2(0,5)$ & - & $98(36,7)$ \\
Escolaridade & & $70(59,8)$ & $1(0,3)$ \\
$\quad$ Até 8 anos de estudo & $238(62,0)$ & $47(40,2)$ & - \\
9 anos e mais & $145(37,8)$ & $1(0,2)$ &
\end{tabular}

eSF: equipes de Saúde da Família; eAB: equipes de Atenção Básica

A Tabela II apresenta a descrição do percentual de avaliação satisfatória (escores $\geq 6,6$ ) dos atributos avaliados pelos usuários de forma geral e segundo o tipo de UBS de referência. Na avaliação do município observou-se que $82,3 \%$ e $93,2 \%$ dos usuários avaliaram positivamente o grau de afiliação e o atributo essencial de primeiro contato (utilização), respectivamente, sendo esses os componentes mais bem avaliados. 
Em relação ao escore essencial, 49,5\% (IC95\%: 44,0\% - 54,4\%) das avaliações foram satisfatórias, enquanto no escore geral foi de $31,8 \%$ (IC95\%: $27,1 \%-36,5 \%)$.

A Tabela II também demonstra que todos os atributos da APS e, respectivamente, os escores essencial e geral, receberam maior percentual de avaliações satisfatórias nas eSF quando comparados às eAB.

Cabe ressaltar que o atributo mais bem avaliado pelos usuários foi o de primeiro contato (utilização). Por outro lado, a orientação comunitária e enfoque familiar foram os atributos com menor percentual de avaliação satisfatória.

A Tabela III apresenta as variáveis explicativas segundo o percentual de avaliação satisfatória (escores $\geq 6,6$ ) dos escores essencial e geral. Segundo as características dos usuários, as variáveis tipo de UBS (eSF e eAB) e escolaridade (até 8 anos ou 9 anos e mais) mostraram significância estatística em relação à proporção de qualidade dos escores essencial e geral, sendo o percentual de avaliação satisfatória maior nas eSF do que nas eAB.

Tabela II - Análise descritiva das avaliações que atingiram o escore de qualidade na Atenção Primária à Saúde (APS) e comparação das avaliações entre o tipo de Unidade Básica de Saúde pelos usuários. Minas Gerais, Brasil, 2016.

\begin{tabular}{lccc}
\hline & \multicolumn{2}{c}{ Proporção (\%) de escores $\geq \mathbf{6 , 6}$} \\
Atributos da APS & $\begin{array}{c}\text { Município } \\
\text { (n=384) }\end{array}$ & $\begin{array}{c}\text { Tipo de Unidade Básica de Saúde } \\
\text { eSF } \\
\text { (n=117) }\end{array}$ & $\begin{array}{c}\text { eAB } \\
\text { (n=267) }\end{array}$ \\
\hline${ }^{*}$ Grau de afiliação & 82,3 & 83,5 & 92,5 \\
Primeiro contato (utilização) & 93,2 & 94,9 & 53,0 \\
Primeiro contato (acesso) & 53,8 & 55,8 & 37,1 \\
Longitudinalidade /atendimento continuado & 44,4 & 61,2 & 57,8 \\
Coordenação (cuidado e sistemas de informação) & 62,5 & 71,2 & 33,1 \\
Integralidade (serviços disponíveis) & 36,3 & 45,0 & 36,5 \\
Integralidade (serviços prestados) & 42,8 & 57,5 & 20,6 \\
Enfoque familiar & 25,0 & 35,1 & 13,6 \\
Orientação comunitária & 17,6 & 27,6 & 44,2 \\
** Escore essencial & 49,5 & 61,5 & 26,2 \\
*** Escore geral & 31,8 & 44,4 & \\
\hline
\end{tabular}

- Grau de afiliação: visa identificar o serviço ou profissional de saúde (médico/enfermeiro) que serve como referência para o cuidado.

* Escore essencial: medido pela soma do grau de afiliação mais escore médio de cada um dos componentes que pertencem aos atributos essenciais, dividido pelo número de componentes.

*** Escore geral: medido pela soma do grau de afiliação mais escore médio dos componentes que pertencem aos atributos essenciais e derivados dividido pelo número total de componentes.

eSF: equipes de Saúde da Família; eAB: equipes de Atenção Básica

Tabela III - Distribuição das variáveis explicativas segundo os escores essencial e geral. Minas Gerais, Brasil, 2016.

\begin{tabular}{lcccc}
\hline \multirow{2}{*}{ Variáveis explicativas } & \multicolumn{2}{c}{ Escore essencial (\%) } & \multicolumn{2}{c}{ Escore geral (\%) } \\
\cline { 2 - 5 } & $\mathbf{2 6 , 6}$ & $\mathbf{p}$ - valor & $\mathbf{2 6 , 6}$ & $\mathbf{p}$ - valor \\
\hline Tipo de Unidade Básica de Saúde & & & & \\
$\quad$ Equipe de Saúde da Família & 61,5 & 0,002 & 44,4 & $<0,001$ \\
$\quad$ Equipe de Atenção Básica & 42,2 & & 26,2 & \\
Sexo & & & & \\
$\quad$ Masculino & 47,3 & 0,658 & 32,4 & 0,905 \\
$\quad$ Feminino & 50,2 & & 31,7 & \\
Idade (anos) & & & \\
$\quad$ Até 40 & 47,2 & 0,443 & 26,8 & 0,277 \\
$\quad$ De 40 a 59 & 48,1 & & 33,1 & \\
$\quad$ 60 e mais & 55,2 & & 36,5 & \\
Situação conjugal & & & \\
$\quad$ Solteiro, divorciado, viúvo & 48,8 & 0,744 & 32,1 & 0,954 \\
$\quad$ União estável & 50,5 & & 31,8 & \\
Escolaridade (anos de estudo) & & & \\
$\quad$ Até 8 & 55,5 & 0,003 & 37,0 & 0,006 \\
$\quad$ 9 e mais & 40,0 & & 23,4 & \\
\hline
\end{tabular}


A Tabela IV mostra a avaliação dos escores essencial e geral satisfatórios, em que as variáveis do tipo de UBS e escolaridade mostraram associação e permaneceram no modelo final da regressão logística. Para a variável tipo de UBS, a chance dos usuários assistidos por eSF atribuírem escore essencial satisfatório aos serviços da APS, foi 2,09 vezes a chance daqueles assistidos por $\mathrm{AB}$. E a chance dos usuários assistidos por eSF atribuírem escore geral satisfatório aos serviços da APS, foi 2,33 vezes a chance daqueles assistidos por eAB.

Quanto à escolaridade, a chance dos usuários com até 8 anos de estudo atribuírem escore essencial satisfatório aos serviços foi 1,94 vezes a chance dos usuários com 9 anos e mais de estudo. $E$ a chance dos usuários com até 8 anos de estudo atribuírem escore geral satisfatório aos serviços foi 2,05 vezes a chance dos usuários com 9 anos e mais de estudo.

Tabela IV - Fatores que influenciam na qualidade do escore essencial e geral da Atenção Primária à Saúde. Minas Gerias, Brasil, 2016.

\begin{tabular}{|c|c|c|c|c|}
\hline \multirow[b]{2}{*}{ Fatores influenciadores } & \multicolumn{2}{|c|}{ Escore essencial } & \multicolumn{2}{|c|}{ Escore geral } \\
\hline & $\begin{array}{l}\text { Razão de chances } \\
\text { (IC 95\%) }\end{array}$ & $p$ valor & $\begin{array}{c}\text { Razão de chances } \\
\text { (IC 95\%) }\end{array}$ & $p$ valor \\
\hline \multicolumn{5}{|l|}{ Tipo de Unidade Básica de Saúde } \\
\hline Equipes de Atenção Básica & 1 & & & \\
\hline Equipes de Saúde da Família & $2,09(1,33-3,28)$ & 0,001 & $2,33(1,47-3,71)$ & $<0,001$ \\
\hline \multicolumn{5}{|l|}{ Escolaridade (anos de estudo) } \\
\hline 9 e mais & 1 & & & \\
\hline Até 8 & $1,94(1,27-2,98)$ & 0,002 & $2,05(1,25-3,24)$ & 0,004 \\
\hline
\end{tabular}

\section{DISCUSSÃO}

Os atributos que alcançaram um percentual considerável de avaliação satisfatória, como o de primeiro contato (utilização), a longitudinalidade e a coordenação (cuidados e sistemas de informação), evidenciam que os indivíduos assistidos por eSF e eAB procuram a UBS como fonte de cuidados, estabelecendo melhor vínculo e confiança e, consequentemente, maior garantia de longitudinalidade do cuidado ${ }^{(18)}$. Segundo o tipo de UBS, as eSF apresentam maior presença e extensão de todos os atributos da APS quando comparadas às eAB.

No entanto, de forma geral, a maioria dos usuários não avaliou bem a presença e extensão dos atributos da APS, denotando que algumas fragilidades podem estar presentes no contexto da APS no cenário em análise. Os atributos que não foram bem avaliados demonstram que os usuários apontam acesso dificultado e demorado à UBS, falta de programas e serviços disponíveis, e desconhecimento das equipes em relação às necessidades do indivíduo, da família e da comunidade. Essa expressão de insatisfação com os serviços indica que a APS, no cenário analisado, apresenta baixo poder de interação com usuários ${ }^{(9,19)}$.

Uma possível explicação para a diferença envolvendo a maior presença e extensão de todos os atributos nas eSF quando comparadas às eAB transpassa a organização do processo de trabalho das equipes. As eSF favorecem a reorientação do processo de trabalho com maior potencial de resolutividade e impacto na situação de saúde da população. É a principal estratégia de qualificação e consolidação da APS, e estrutura-se nas reais necessidades da população e no contato permanente com o território ${ }^{(1)}$.

A discussão de casos em reuniões de equipe, a realização de visitas domiciliares, a presença do agente comunitário de saúde (ACS), considerado elo fundamental na mediação entre a eSF e comunidade, o conhecimento acerca dos problemas de saúde da população e o leque de serviços prestados em caráter biopsicossocial influenciam na maior presença e extensão dos atributos em eSF. Além disso, o contato entre os profissionais de saúde e usuários, assim como o estabelecimento da referência e contrarreferrência quando encaminhado ao especialista, são fatores que se destacam em eSF quando comparadas às $\mathrm{eAB}{ }^{(20)}$.

No que se refere a cada atributo da APS avaliado, embora exista a interação dos usuários com os serviços mediante um novo problema de saúde ou episódio de um mesmo problema, o baixo percentual de avaliação satisfatória do atributo de primeiro contato (acesso) retrata dificuldades no acesso à UBS. Diversos fatores podem estar relacionados a essas limitações, como a localização da unidade, os horários de funcionamento, a disponibilidade e a conveniência dos serviços ${ }^{(7)}$.

Cabe ressaltar que o atributo essencial de primeiro contato (utilização) contribuiu consideravelmente na qualidade da avaliação do escore essencial em eAB, uma vez que o restante dos atributos não obtiveram percentuais de avaliação satisfatória significativos. Como já mencionado, as eSF receberam um maior percentual de avaliação satisfatória em relação a todos os atributos da APS quando comparadas às eAB, tal fato influenciou o melhor 
desempenho das eSF nos escores essencial e geral. Avaliação positiva do escore essencial em eSF refere-se à forma como as eSF organizam as ações e serviços, mas também demonstram a necessidade de fortalecimento( ${ }^{(4)}$.

Outro desafio encontrado no presente estudo refere-se à falta de programas e serviços disponíveis para que o indivíduo receba atenção do ponto de vista biopsicossocial, bem como de ações de promoção, proteção, tratamento e reabilitação. Esse fato demonstra que ainda existem lacunas no processo de trabalho das equipes, na qualificação profissional e na organização das RAS, além de indicar a persistência do modelo biomédico centrado na doença(19,21). A disposição adequada e o reconhecimento dos serviços e recursos disponibilizados pressupõe a construção de um diagnóstico situacional da área de abrangência e de um planejamento das ações voltadas para as necessidades de saúde da população( ${ }^{(21)}$.

Os atributos derivados, enfoque familiar e orientação comunitária, apresentaram baixo percentual de avaliação satisfatória, influenciando, consequentemente, na qualidade da avaliação do escore geral. Apesar do enfoque familiar e da orientação comunitária aparecerem com melhor desempenho nas eSF quando comparados às eAB, esses apresentam percentual de avaliação satisfatória aquém do ideal, considerando os princípios e diretrizes da ESF, reafirmando que, tanto em eSF quanto em eAB, há um conhecimento insuficiente dos profissionais em relação às necessidades de saúde da população, não correlacionando o atendimento do indivíduo e da família à realidade ${ }^{(18)}$.

A orientação à APS com características como enfoque familiar e orientação comunitária constitui um novo paradigma para o SUS. Considera-se a dificuldade dos serviços em integrarem a família e a comunidade no processo de cuidado e a falta de conhecimento da comunidade acerca das atividades realizadas nas UBS como fatores que podem fortalecem a cultura da assistência curativista e centrada na doença ${ }^{(22)}$.

Um serviço de saúde orientado à APS a partir da presença desses atributos favorece o planejamento e execução de ações, assim como o fortalecimento do vínculo entre serviço de saúde, indivíduo, família e comunidade, gerando impacto para o SUS como política de saúde abrangente e universal. As equipes e usuários devem permanecer aliados no desenvolvimento das ações de prevenção, promoção e recuperação dos agravos, objetivando a autonomia e o enfrentamento dos determinantes sociais da saúde ${ }^{(13)}$. Dessa forma, o cuidado se torna mais efetivo, centrado nas necessidades de saúde da população e, consequentemente, acarreta melhoria nos indicadores de saúde e na promoção da saúde da população(22,23).

Além disso, a escolaridade também foi um fator que influenciou a qualidade dos escores essencial e geral da APS. Ao contrário do que foi demonstrado neste estudo, a maior escolaridade está associada a uma melhor percepção de desempenho da APS, podendo estar relacionada à capacidade do usuário obter, compreender e comunicar informações básicas em saúde ${ }^{(24,25)}$. Um maior nível de escolaridade pode proporcionar maior compreensão do que é orientado, bem como propiciar o levantamento de questões relativas à saúde que envolvem o bemestar $\mathrm{e}$ aspectos preventivos ${ }^{(22)}$.

Mesmo com as eSF apresentando melhores resultados quando comparadas às eAB, a coexistência de diferentes modelos assistenciais torna os serviços da APS complementares ou concorrentes ${ }^{(26)}$. Os diferentes modelos assistenciais ainda presentes demonstram fragilidades no potencial da APS de promover mudanças nas práticas clínico-assistenciais, evidenciando que as ações continuam predominantemente centradas em atividades de tratamento e reabilitação de doenças e que há deficiências no processo de trabalho das equipes ${ }^{(27)}$. Fazem-se necessárias mudanças de atitude e nas práticas de gestores, e um olhar integral para o indivíduo, família e comunidade, com concepções macro e micropolíticas, a fim de garantir efetividade dos serviços da APS e maior presença e extensão dos atributos ${ }^{(23)}$.

As limitações do presente estudo envolvem o fato de ter sido realizado em um único município, porém cabe ressaltar que se deve discutir a avaliação de serviços considerando a realidade local. Há, ainda, dificuldades inerentes ao delineamento do estudo que poderiam ser minimizadas com base em estudos complementares com abordagem qualitativa. Cabe ressaltar que, apesar das fragilidades apresentadas, existem pontos a serem destacados, como 0 reconhecimento da UBS pelo usuário como a principal porta de entrada do sistema de saúde. Os desafios a serem superados envolvem, principalmente, aqueles relacionados ao processo de trabalho das equipes. Além disso, deve-se investir em estratégias de redirecionamento das práticas voltadas às necessidades de saúde da população, ao enfoque familiar e à participação comunitária.

\section{CONCLUSÃO}

Conclui-se que, na visão dos usuários investigados, a eSF apresentou melhor potencial para a presença e extensão dos atributos/componentes, reforçando o papel de destaque dessa estratégia como principal arranjo organizacional de APS no cenário nacional. 
Cabe ressaltar a importância da avaliação da qualidade dos serviços da APS na visão de usuários a fim de promover melhorias nos serviços prestados. Sugere-se a utilização do PCATool-Brasil, versão reduzida, para avaliação rotineira em UBS.

\section{CONFLITOS DE INTERESSE}

Não existem conflitos de interesses.

\section{CONTRIBUIÇÕES}

Todos os autores contribuíram com a elaboração e delineamento do estudo; a aquisição, análise e interpretação de dados; a redação e/ou revisão do manuscrito.

Estudo baseado na dissertação: Avaliação da presença e extensão dos atributos da Atenção Primária à Saúde em um município da região centro-oeste de Minas Gerais. Dissertação (Mestrado) - Programa de Pós-graduação Mestrado Acadêmico em Enfermagem da Universidade Federal de São João del-Rei (UFSJ), 2018, 91 p.

\section{REFERÊNCIAS}

1. Brasil. Portaria $n^{\circ} 2.436$, de 21 de setembro de 2017. Aprova a Política Nacional de Atenção Básica, estabelecendo a revisão de diretrizes para a organização da Atenção Básica, no âmbito do Sistema Único de Saúde (SUS). Ministério da Saúde 21 de setembro de 2017.

2. O'Malley AS, Rich EC. Measuring Comprehensiveness of Primary Care: Challenges and Opportunities. J Gen Intern Med [Internet]. 2015 [acesso em 2017 Nov 11];30(suppl 3):S568-75p. Disponível em: https://www.ncbi. nlm.nih.gov/pmc/articles/PMC4512967/?tool=pubmed

3. Ministério da Saúde (BR). Sala de Apoio à Gestão Estratégica [Internet]. [acesso em 2018 Mar 20]. Disponível em: http://sage.saude.gov.br/

4. Silva SA, Fracolli LA. Avaliação da assistência à criança na Estratégia de Saúde da Família. Rev Bras Enferm [Internet]. 2016 [acesso em 2018 Abr 09];69(1):47-53. doi:10.1590/0034-7167.2016690107i

5. Arantes LJ, Shimizu HE, Merchán-Hamann E. Contribuições e desafios da Estratégia Saúde da Família na Atenção Primária à Saúde no Brasil: revisão da literatura. Cienc Saude Colet [Internet]. 2016 [acesso em 2017 Nov 07];21(5):1499-1509. doi:10.1590/1413-81232015215.19602015

6. Bulgareli J, Cortellazzi KL, Ambrosano GMB, Meneghim MC, Faria ET, Mialhe FL, et al. A resolutividade em saúde bucal na atenção básica como instrumento para avaliação dos modelos de atenção. Cienc Saude Colet [Internet]. 2014 [acesso em 2017 Ago 20];19(2):383-91. doi:10.1590/1413-81232014192.20102012

7. Starfield B. Atenção Primária: equilíbrio entre necessidades de saúde, serviços e tecnologia. Brasília: UNESCO; 2002.

8. Starfield B, Xu J, Shi L. Validating the Adult Primary Care Assessment Tool. J Fam Pract [Internet]. 2001 [acesso em 2017 Ago 10];50(2):161-75. Disponível em: http://www.mdedge.com/jfponline/article/60464/ validating-adult-primary-care-assessment-tool

9. Prates ML, Machado JC, Silva LS, Avelar PS, Prates LL, Mendonça ET, et al. Desempenho da Atenção Primária à Saúde segundo o instrumento PCATool: uma revisão sistemática. Cienc Saude Colet [Internet]. 2017 [acesso em 2018 Nov 13];22(6):1881-93. doi:10.1590/1413-81232017226.14282016

10. Cardoso AVL, Chain APN, Mendes RIP, Ferreira EF, Vargas AMD, Martins AMEBL, et al. Assessment of the management of the family health strategy via the tool Assessment for Quality Improvement in municipalities of minas Gerais, Brazil. Cienc Saude Colet [Internet]. 2015 [acesso em 2017 Nov 19];20(4):1267-84. doi:10.1590/1413-81232015204.01832014

11. Reis RS, Coimbra LC, Silva AAM, Santos AM, Alves MTSSB, Lamy ZC, et al. Acesso e utilização dos serviços na Estratégia Saúde da Família na perspectiva dos gestores, profissionais e usuários. Cienc Saude Colet [Internet]. 2013 [acesso em 2018 Abr 09];18(11):3321-31. doi:10.1590/S1413-81232013001100022 
12. Pinto EP Jr, Cavalcante JLM, Sousa RA, Morais APP, Silva MGC. Análise da produção científica sobre avaliação, no contexto da saúde da família, em periódicos brasileiros. Saúde Debate [Internet]. 2015 [acesso em 2018 Jan 15];39(104):268-78. doi:10.1590/0103-110420151040226

13. Paula WKAS, Samico IC, Caminha MFC, Batista M Filho, Figueirôa JN. Orientação comunitária e enfoque familiar: avaliação de usuários e profissionais da estratégia saúde da família. Cad Saude Colet [Internet]. 2017 [acesso em 2018 Mar 10];25(2):242-48. doi:10.1590/1414-462x201700020113

14. Grimm S, Padilha A, Bassichetto KC, Lira M. Dezesseis anos de monitoramento em saúde na atenção primária em uma grande metrópole das Américas. Rev Panam Salud Publica [Internet]. 2018 [acesso em 2019 Jul 30];42:e183. doi:10.26633/RPSP.2018.183

15. IBGE. Divinópolis [Internet]. [2018] [acesso em 2018 Mar 10]. Disponível em: https://cidades.ibge.gov.br/ brasil/mg/divinopolis/panorama

16. Oliveira MMC, Harzheim E, Riboldi J, Duncan BB. PCATool-ADULTO-BRASIL: uma versão reduzida. Rev Bras Med Fam Comunidade [Internet]. 2013 [acesso em 2017 Jul 18];8(29):256-63. doi:10.5712/ rbmfc8(29)823

17. Ministério da Saúde (BR). Manual do instrumento de avaliação da atenção primária à saúde: primary care assessment tool pcatool - Brasil. Brasília: Ministério da Saúde; 2010.

18. Oliveira VBCA, Veríssimo MLÓR. Children's health care assistance according to their families: a comparison between models of Primary Care. Rev Esc Enferm USP [Internet]. 2015 [acesso em 2017 Jul 18];49(1):30-6. doi:10.1590/S0080-623420150000100004

19. Mesquita M Filho, Luz BSR, Araújo CS. A Atenção Primária à Saúde e seus atributos: a situação das crianças menores de dois anos segundo suas cuidadoras. Cienc Saude Colet [Internet]. 2014 [acesso em $2018 \mathrm{Abr}$ 09];19(7):2033-46. doi:10.1590/1413-81232014197.17322013

20. Martins JS, Abreu SCC, Quevedo MP, Bourget MMM. Estudo comparativo entre Unidades de Saúde com e sem Estratégia Saúde da Família por meio do PCATool. Rev Bras Med Fam Comunidade [Internet]. 2016 [acesso em 2018 Abr 09];11(38):1-13. doi:10.5712/rbmfc11(38)1252

21. Shimazaki ME. A Atenção Primária à Saúde. Belo Horizonte: ESPMG; 2009.

22. Araújo JP, Viera CS, Toso BRGO, Collet N, Nassar PO. Assessment of attributes for family and community guidance in the child health. Acta Paul Enferm [Internet]. 2014 [acesso em 2018 Jan 06];27(5):440-6. doi:10.1590/1982-0194201400073

23. Reichert APS, Leônico ABA, Toso BRG, Santos NCCB, Vaz EMC, Collet N. Orientação familiar e comunitária na Atenção Primária à Saúde da criança. Cienc Saude Colet [Internet]. 2016 [acesso em 2018 Jan 06];21(1):119-27. doi:10.1590/1413-81232015211.05682014

24. Leão CDA, Caldeira AP, Oliveira MMC. Atributos da atenção primária na assistência à saúde da criança: avaliação dos cuidadores. Rev Bras Saúde Mater Infant [Internet]. 2011 [acesso em 2018 Jan 15];11(3):32333. doi:10.1590/S1519-38292011000300013

25. Augusto DK, Lima-Costa MF, Macinko J, Peixoto SV. Fatores associados à avaliação da qualidade da atenção primária à saúde por idosos residentes na Região Metropolitana de Belo Horizonte, Minas Gerais, 2010. Epidemiol Serv Saude [Internet]. 2019 [acesso em 2019 Jul 30];28(1):e2018128. doi:10.5123/S1679-49742019000100017

26. Silva AS, Baitelo TC, Fracolli LA. Avaliação da atenção primária à saúde: a visão de usuários e profissionais sobre a estratégia de saúde da família. Rev Latinoam Enferm [Internet]. 2015 [acesso em 2018 Abr 09];23(5):979-87. doi:10.1590/0104-1169.0489.2639

27. Fertonani HP, Pires DEP, Biff D, Scherer MDA. Modelo assistencial em saúde: conceitos e desafios para a atenção básica brasileira. Cienc Saude Colet [Internet]. 2015 [acesso em 2017 Out 21];20(6):1869-78. doi:10.1590/1413-81232015206.13272014 


\section{Endereço para correspondência:}

Anna Gabryela Sousa Duarte

Universidade Federal de São João del-Rei - UFSJ

Rua Sebastião Gonçalves Coelho, 400

Bairro: Chanadour

CEP: 35501-296 - Divinópolis - MG - Brasil

E-mail: annagsd@hotmail.com

Como citar: Duarte AGS, Gontijo TL, Guimarães EAA, Cavalcante RB, Belo VS, Silva GS. Fatores associados ao desempenho de serviços da atenção primária à saúde. Rev Bras Promoç Saúde. 2019;32:8843. 